\title{
Implementation and validation of a strain rate dependent model for carbon foam
}

\author{
G. Janszen \& P. G. Nettuno \\ Politecnico di Milano, Dipartimento di Ingegneria Aerospaziale, Italy
}

\begin{abstract}
Carbon foam showed good ballistic performances for relatively small fragment impacts: low density samples $\left(0.56 \mathrm{~g} / \mathrm{cm}^{3}\right.$ and $\left.0.24 \mathrm{~g} / \mathrm{cm}^{3}\right)$ were able to stop and in some cases hold a $5 \mathrm{~mm}$ diameter stainless steel sphere shot at a speed up to $240 \mathrm{~m} / \mathrm{s}$ by a compressed air gun.

The results were used to calibrate and benchmark an Ls-Dyna model which had to be based only on a few and easy-to-measure material parameters. Therefore, performing only static compressive loading characterization tests, a suitable cellular Ls-Dyna material model was chosen. To justify the promising energy dissipation results, which cannot only be due to the static performances, a strain rate dependency was supposed. Based on ceramic materials which have inhomogeneities of the same size of the foam pores, a strain rate law typical of these was applied.

Similar relations were applied to both the foams, and a calibrating coefficient was made on a single impact velocity test. The same model was then used to reproduce the impact at different impact velocities and very good agreement between experimental results and simulation was achieved.
\end{abstract}

Keywords: carbon foam, ballistic impact, strain-rate effect.

\section{Introduction}

Foams, both in the form of natural ones as well as engineered, have been used widely in very different fields for a long time. The applications vary from packaging shock mitigation, to lightweight structure for the automotive and aerospace fields. It is therefore possible to find comprehensive references covering general characteristics and illustrating the fields of application [1].

However, carbon foams represent a relatively novel group of materials, for which a widely available characterization database is not easily accessible. The 
reasons to investigate them are not only justified by the need to cover this lack of knowledge but by the chance to use them for different applications. They can be used as catalysts, electrodes, while the high surface area combined with the nature of pure glassy carbon make carbon foam well suited for filtration systems and for fuel cells [2]. There is no specific application suggestion or example available as structural component: there are just a few characterization investigations for various configurations. They move from simple sandwich panels used in quasi-static test [3] to studies about hypervelocity impact protection for space vehicles [4]. Moreover, the similar coefficient of thermal expansion suggests the use of carbon foams for carbon composites sandwich panels.

Based on these reasons and on the fact that usually the cellular structures allow better energy dissipation performances in respect of the full dense solid [1], a complete investigation program has been developed. The program has been thought to understand and model the mechanical behaviour of these materials in quasi static and high strain-rate regimes. The ballistic tests showed that in spite of the limited fracture toughness, these foams were able to dissipate in an efficient way the kinetic energy of relatively small spherical stainless steel bullets. One interesting feature was that even if the foams showed a quasi static fragile behaviour, after the high speed impacts the dimensions of the damage were limited. The crushing mechanism involved at the micromechanical scale can provide for bullet entrapment passing through impact velocity reduction and rebound control. This is of particular interest for all those protective applications in which not only target trespassing is considered fatal but protection from bullet rebound prevents catastrophic failures.

In this paper we present an overview of the performed low strain rate characterization campaign of two carbon foams with particular interest for the confined compression which leads to uniaxial strain.

There was no chance to test and measure the dynamic properties of the materials under high strain rate loading condition, therefore a phenomenogical relationship between compressive strength $\sigma$ and strain rate $\dot{\varepsilon}$ [5] was used to model the material behaviour. This was then implemented in an Ls-Dyna material model to simulate the ballistic experiments. This relationship is generally used for the ceramics and is based on their micro-cracks evolution, which have sizes comparable with the cells dimensions in the tested carbon foams. This is reported in eqn.(1).

$$
\sigma=\sigma_{c 0}+B \cdot \dot{\varepsilon}^{N}
$$

The exponent $\mathrm{N}$ is $1 / 3$ which is a common value for these materials.

\section{Quasi-static mechanical characterization}

A complete quasi-static experimental measurement campaign was performed to better understand material behaviour and its characteristics. In the following subsections an overview of the results will be presented. 


\subsection{Uniaxial compression}

Typical measurements with an MTS machine under a mono-axial stress compressive condition, showed a highly brittle mechanical behaviour of both investigated foams. During the tests, performed in accordance with the [7] norm at a speed of $0.25 \mathrm{~mm} / \mathrm{min}$, the specimens reached ultimate failure with very low deformation values. They showed a sudden loss in load bearing capability for strains value of about $1.5 \%$. The cracks evolved in a nearly instantaneous and instable manner with the form of large and long separations along the load application axis.

Examples of specimen failure and stress-strain curve are reported in Figure 1.
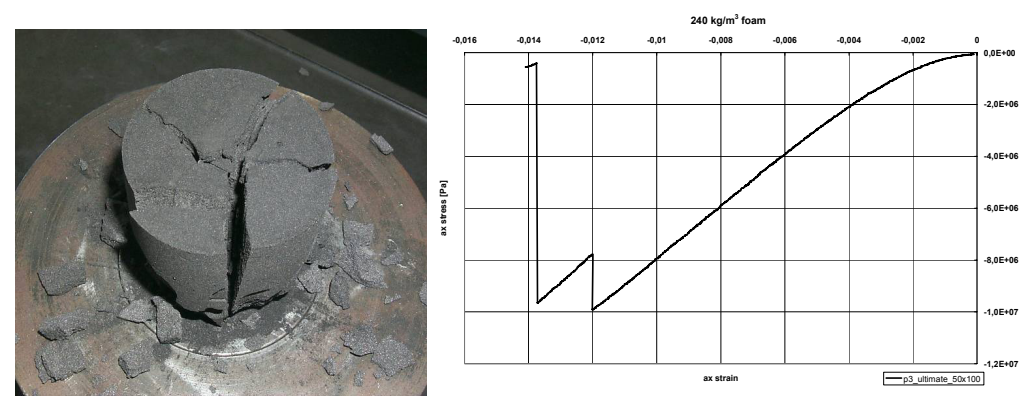

Figure 1: $\quad$ Example of cylindrical specimen failure under 1-D stress loading condition and the correspondent stress-strain curve.

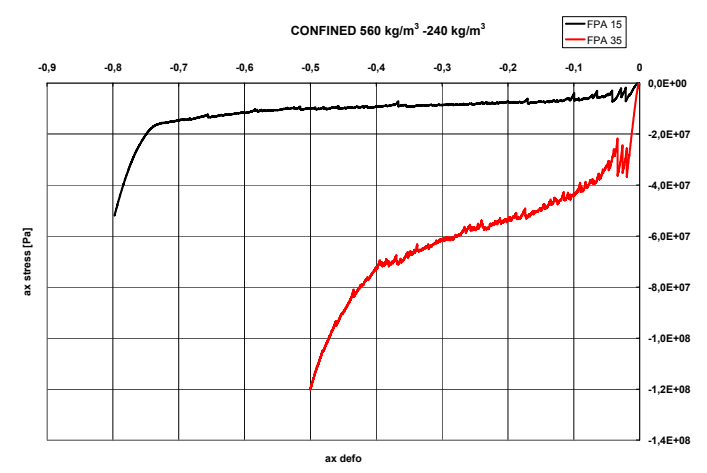

Figure 2: Stress-strain curves measured under lateral confinement for uniaxial strain loading conditions.

\subsection{Confined compression}

Confined compression experiments demonstrated a dramatic change in material behaviour. This meant mostly a wide continuation of the load capability up to very large strains. As it is possible to notice in Figure 2, instead of a brittle 
nature with a sudden load decay, the lateral confinement restricted the evolution of the cracks in the specimen. This is reflected in a three zones stress strain curve, composed by: a first elastic portion which copes mainly the unconfined results, a second stage which for the less dense foam is basically a wide constant stress plateau while the denser one still exhibits a noticeable stress increment. This part of the curves is what is referred as brittle crushing plateau [1] and it is the main mechanism which absolves energy dissipation and shock mitigation.

The last portion is what corresponds to the densification strain, limit in which the porosity of the material has been filled by the broken fragments and ligaments that constitute the cellular structure of the foam. This stage, which happens for strain values of 0.8 and 0.5 for the less and more dense foam, corresponds to carbon dust compression with a steep rise in the stress value.

\section{Computational analyses}

The simulation of the ballistic impact was computed using LS-Dyna 971 r. 3.2.1 in double precision mode. This is a commercial state of the art software, derived from the public software DYNA 3D which was developed by the "Lawrence Livermore National Laboratories" in the seventies. It is particularly suited for transient non linear dynamics mechanical interaction modelling such as impacts, crashes and penetrations.

Model development followed an iterative revision process while the understanding of the experimental evidences was in progress.

An overview of the material models which have been chosen to describe the dynamic response of the involved materials is presented in Section 3.2 and its subsections.

\subsection{Problem definition}

The impact problem analyzed in the present work consists of a spherical steel impactor with a diameter of nominal $5 \mathrm{~mm}$, with a mass of 0.5 grams colliding at different speeds a solid block of carbon foam. The values of the velocity were in accordance with the experiments: $240 \mathrm{~m} / \mathrm{s}, 210 \mathrm{~m} / \mathrm{s}, 75 \mathrm{~m} / \mathrm{s}$, and perpendicular to the foam surface. The carbon foam surface hit is a square with a side of $50 \mathrm{~mm}$ while the depth of the block is $40 \mathrm{~mm}$. The bullet impacts the carbon foam in the top face central point: a condition close to reality but not always verified in the ballistic experiments. The whole model is composed of 8 nodes hexahedrons solids with single integration point. Different meshes were used to model the domain both for target and for impactor, allowing various combinations of refinements of the two model parts.

\subsection{Material modelling}

There are basically two key points regarding the identification of the material characteristics in the model: the first one is the use of the information deriving by the confined compression test, which allows to consider the material like an effective foam behaving one. The second refers to the extension, application and 
calibration of the micromechanical models relating compressive strength and high strain rate commonly used for ceramics. These latter ones exhibit a brittle compressive behaviour similar to the carbon foam under quasi static compression. In addition to this, they show an evident and strong raise of the compressive strength in case of dynamic loading process. This property, which is generically common for the foams too, justifies the measured ballistic performances. It is worth to notice that the approach of considering the compressive confined behaviour of similar materials involved in ballistic impact has been already used [6].

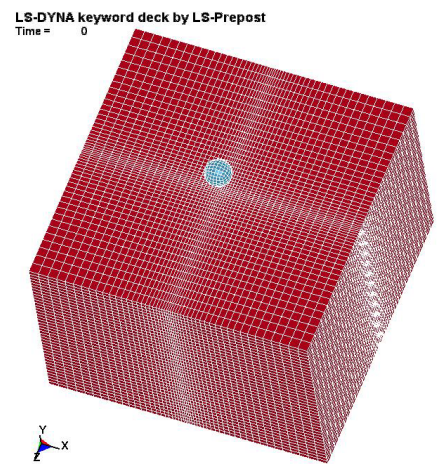

Figure 3: Finite element model of the ballistic experiments presented in the present work.

\subsubsection{Steel bullet}

Considering the fact that the spherical bullet did not undergo any significant deformation due to the impact with the target, and that the elastic modulus is two orders of magnitude higher than the one of the foams a simple model to limit computational costs was used for it.

The characteristics were the ones of the AISI 4130 steel as reported by the MIL-HDBK 5H 1/12/98 (Table 1).

Table 1: $\quad$ Bullet material data.

\begin{tabular}{c|c}
\hline \multicolumn{2}{c}{ AISI 4130 Steel - MIL HDBK 5H 1/12/98 } \\
\hline Density $\left[\mathrm{kg} / \mathrm{m}^{3}\right]$ & 7833 \\
\hline $\mathrm{E}[\mathrm{GPa}]$ & 200 \\
\hline $\mathrm{G}[\mathrm{GPa}]$ & 76 \\
\hline
\end{tabular}

The material model is the number 1 (MAT_ELASTIC) in the Ls-Dyna library [8], and is used for isotropic elastic materials, needing only density, Young's modulus and Poisson ratio.

\subsubsection{Carbon foam target}

The procedure to individuate the correct material relied first on the definition of the material model in the Ls-Dyna library which could include the peculiarities 
showed by the foams in the experimental campaign. These are represented by stress-strain curves input by points, and different behaviours depending on instantaneous strain-rate value. Referring to the measured static confined compression curves different stress-strain relationships, varying the B parameters of eqn.(1), were computed covering a range of strain rate values. Once a good agreement between experimental damage in the target due to the impact and the value resulting from the analysis with the standard mesh was reached the $\mathrm{B}$ parameter was fixed and the computations for the other impacts speed were performed.

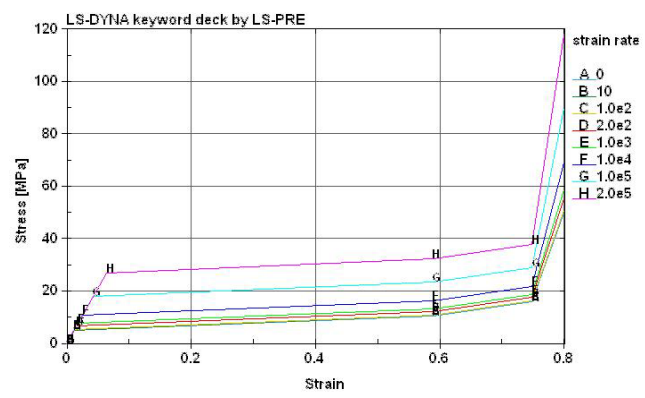

Figure 4: Stress-Strain curves used in the computations for the $240 \mathrm{~kg} / \mathrm{m}^{3}$ foam.

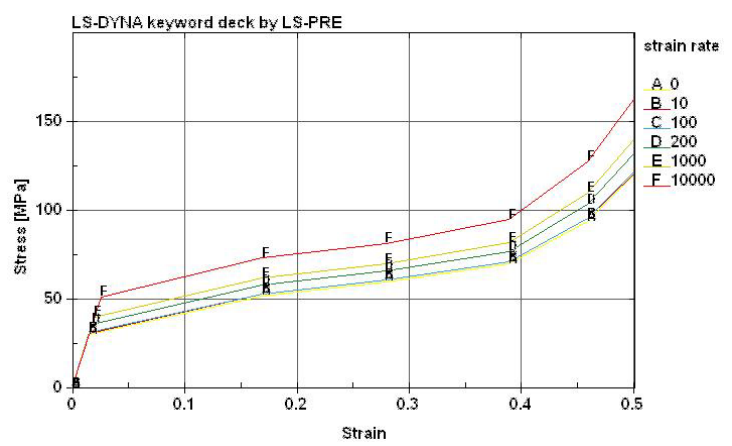

Figure 5: Stress-Strain curves used in the computations for the $560 \mathrm{~kg} / \mathrm{m}^{3}$ foam.

Deep considerations and trials lead to material kind 83 "Mat Fu Chang Foam". It is possible to find interesting application of this model in the automotive [9] and in the aerospace [10] fields. To include the fragile behaviour, material 83 has been completed by an erosion criteria as a limit on the maximum principal strain which corresponds to the value identified as the densification strain. In fact, during the experimental tests, once the maximum strain was reached, the foams were transformed completely in dust. At this stage we considered that this carbon dust was free to evacuate on the back of the bullet 
and/or through the pores of the target. Therefore once the maximum principal strain gets to the limit the corresponding element is deleted and a new contact surface is defined by the "contact eroding surface to surface" algorithm chosen.

\subsubsection{Low density carbon foam target}

For this foam the parameter B resulted 0.28. This means that the stress strain curves used for compression were the ones reported in Figure 4. The erosion value for the principal strain was 0.8 .

\subsubsection{High density carbon foam target}

For this foam the parameter B resulted 1. This lead to the stress-strain curves represented in Figure 5. The limit for erosion for the principal strain is 0.5 .

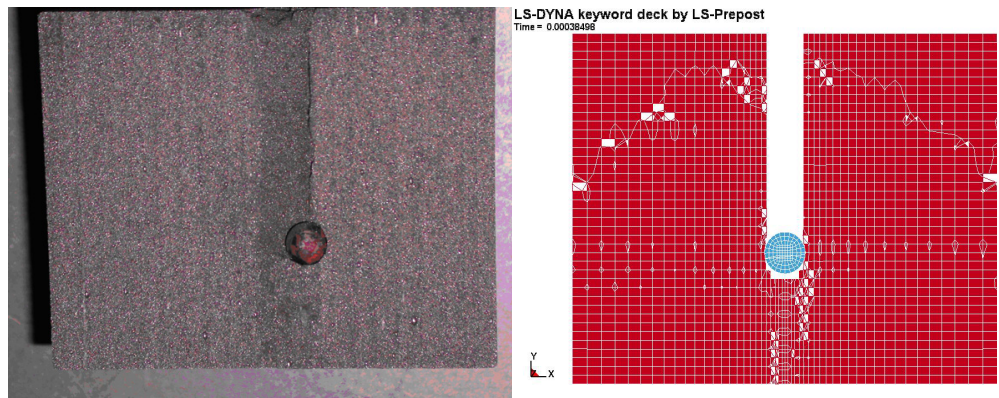

Figure 6: The model (on the right) represented correctly the uniaxial strain resulted in the ballistic experiment.

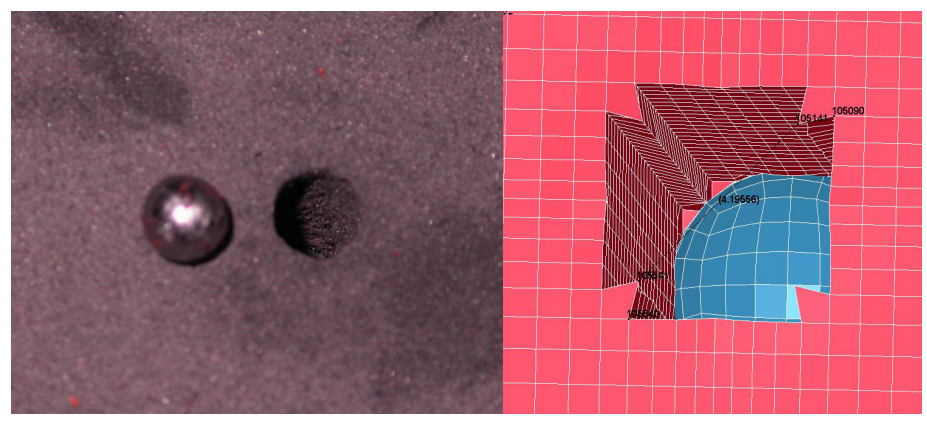

Figure 7: Comparison between test sample and simulation for impact against the light foam at $240 \mathrm{~m} / \mathrm{s}$.

\section{Validation results and discussion}

The type and extent of the damage were very well represented by the simulation which caught the uniaxial deformation process that was highlighted by the ballistic experiments. The shape and the size of the holes were always close to 
the bullet ones without any significant propagation, although the fragile nature of the material could potentially involve cracking instabilities.

As represented in Figures 6,7 and 8, the macro aspects of the impacts were respected for both the foams densities.

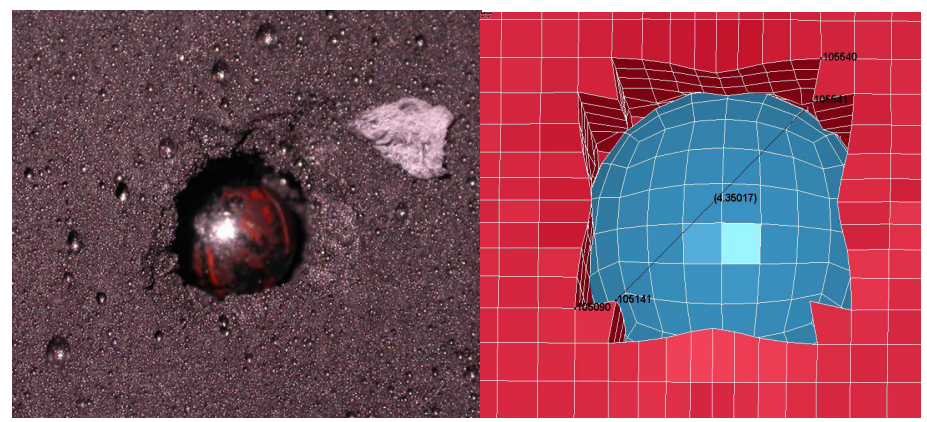

Figure 8: Comparison between test sample and simulation for impact against the denser foam at $240 \mathrm{~m} / \mathrm{s}$.

Once the relationship between strain rate and compressive strength was set, only the impact speeds were varied. The results were absolutely satisfactory in both cases as reported in Table 2 .

Table 2: Comparison of the holes depth value between computations and experimental results. The boxed cells are the ones used to calibrate the compressive strength - strain rate relation.

\begin{tabular}{c|c|c|c}
\hline & $\begin{array}{c}\text { Impact Velocity } \\
{[\mathbf{m} / \mathbf{s}]}\end{array}$ & $\begin{array}{c}\text { Standard mesh }- \\
\text { hole depth [mm] }\end{array}$ & $\begin{array}{c}\text { Experimental hole depth } \\
{[\mathbf{m m}]}\end{array}$ \\
\hline \multirow{2}{*}{$\begin{array}{c}240 \mathrm{~kg} / \mathrm{m}^{3} \\
\text { foam }\end{array}$} & 75 & 4.3 & 4 \\
\cline { 2 - 4 } & 210 & 23 & 22 \\
\cline { 2 - 4 } & 240 & 28 & 28 \\
\hline $560 \mathrm{~kg} / \mathrm{m}^{3}$ & 210 & 8 & 8 \\
\cline { 2 - 4 } foam & 240 & 8.9 & 9 \\
\hline
\end{tabular}

\subsection{Mesh sensitivity}

Several meshes of both target and impactor were used to perform computations once the material curves were set, basing on the standard mesh results.

In Tables 3 and 4 the main features of the target and bullet meshes are respectively reported.

All target meshes were designed with a progressive refinement of the elements size in the central area which is involved by the impact. This has been done to understand the effect of the element size perpendicular to the impact speed and its thickness, avoiding excessive elongation ratio in the velocity direction.

In a consistent way, the bullet meshes were refined dimensioning the elements to assure best interaction between the parts involved in the contact. It 
Table 3: $\quad$ Target meshes main features.

\begin{tabular}{c|c|c|c|c}
\hline $\begin{array}{c}\text { Mesh } \\
\text { kind }\end{array}$ & $\begin{array}{c}\text { Number of } \\
\text { elements on the } \\
\text { impact plane }\end{array}$ & $\begin{array}{c}\text { Central element } \\
\text { diagonal }[\mathbf{m m}]\end{array}$ & $\begin{array}{c}\text { Elements thickness } \\
{[\mathbf{m m}]}\end{array}$ & $\begin{array}{c}\text { Total } \\
\text { number of } \\
\text { elements }\end{array}$ \\
\hline standard & 2.500 & 0.49 & 1 & 100.000 \\
\hline 2 & 3.800 & 0.28 & 0.6 & 252.000 \\
\hline 4 & 1.600 & 0.6 & 0.5 & 128.000 \\
\hline
\end{tabular}

Table 4: $\quad$ Bullet meshes main features.

\begin{tabular}{c|c|c}
\hline Mesh kind & $\begin{array}{c}\text { Element projected diagonal } \\
{[\mathbf{m m}]}\end{array}$ & Total number of elements \\
\hline standard & 0.49 & 864 \\
\hline 3 & 0.3 & 6912 \\
\hline
\end{tabular}

must be noticed that the projected diagonals are the dimensions which are correlated with the size of the elements of the impact area on the target.

Computations showed an high stability concerning mesh sensitivity. The maximum difference reached is of $7 \%$ in the case of target 4 vs. bullet 3 using as baseline the standard case as reported in Table 5. Big differences in elements size did not involve dramatic changes in results: It is worth to notice that target mesh 2 presents half size elements on the impact face compared with the standard case. In spite of this the results showed to be consistent. Comparing the standard target mesh and the mesh 4, the ratio between thickness and the central element diagonal passes from 2 to 1 . This seems to influence slightly the material stiffness. Due to the bias of the mesh on the top surface anyhow this ratio is respected just for the central area due to the constant thickness value of them.

Table 5: $\quad$ Low density foam computations results summary.

\begin{tabular}{c|c|c|c|c|c}
\hline $\begin{array}{c}\text { Impact } \\
\text { Velocity } \\
{[\mathbf{m} / \mathbf{s}]}\end{array}$ & $\begin{array}{c}\text { Standard } \\
\text { mesh - hole } \\
\text { depth [mm] }\end{array}$ & $\begin{array}{c}\text { Bullet 3 vs } \\
\text { Target std - } \\
\text { [mm] }\end{array}$ & $\begin{array}{c}\text { Bullet 3 vs } \\
\text { target 2 - } \\
\text { [mm] }\end{array}$ & $\begin{array}{c}\text { Bullet 3 vs } \\
\text { target 4 } \\
\text { [mm] }\end{array}$ & $\begin{array}{c}\text { Experimental } \\
\text { hole depth } \\
\text { [mm] }\end{array}$ \\
\hline 75 & 4.3 & 4.4 & 4.5 & 4.4 & 4 \\
\hline 210 & 23 & 22 & 22.3 & 21.5 & 22 \\
\hline 240 & 28 & 27 & 27.4 & 26 & 28 \\
\hline
\end{tabular}

Table 6: High density foam computations results summary.

\begin{tabular}{c|c|c|c|c|c|c}
\hline $\begin{array}{c}\text { Impact } \\
\text { Velocity } \\
{[\mathbf{m} / \mathbf{s}]}\end{array}$ & $\begin{array}{c}\text { Standard } \\
\text { mesh - } \\
\text { hole depth } \\
{[\mathbf{m m}]}\end{array}$ & $\begin{array}{c}\text { Bullet std } \\
\text { vs target } \\
\mathbf{2}-[\mathbf{m m}]\end{array}$ & $\begin{array}{c}\text { Bullet 3 } \\
\text { vs Target } \\
\text { std -[mm] }\end{array}$ & $\begin{array}{c}\text { Bullet 3 } \\
\text { vs target } \\
\mathbf{2 -}[\mathbf{m m}]\end{array}$ & $\begin{array}{c}\text { Bullet 3 } \\
\text { vs target } \\
\mathbf{4} \\
{[\mathbf{m m}]}\end{array}$ & $\begin{array}{c}\text { Experimental } \\
\text { hole depth } \\
{[\mathbf{m m}]}\end{array}$ \\
\hline 210 & 8 & 7.5 & 8 & 7.7 & 7.2 & 8 \\
\hline 240 & 8.9 & 9.1 & 9.1 & 9.4 & 8.5 & 9 \\
\hline
\end{tabular}




\section{Conclusions}

A complete experimental investigation in quasi-static compressive stress-strain condition allowed us to understand mechanical behaviour of low and high density carbon foams. In lateral confinement conditions the foam showed a wide brittle crushing plateau which gives the same stress-strain curve shape of an elastic - plastic behaving material. This part of the stress-strain curve shows a very low strain hardening coefficient for the low density foam and a noticeable one for the high density one. The modulus of elasticity was not modified by the confinement. Passing from uniaxial stress to uniaxial strain, the failure evolution changed without influencing the elastic behaviour. Therefore under confinement multiaxial loading condition, these foams present energy absorption much higher than in the case of simple compression, due to the extend crushing plateau.

In ballistic experiments with small fragments (about 1/10 of target thickness) the foam experienced confined compression failure, associated with very limited failure area extension. Even if the extended plateau region allows good energy dissipation properties, this was not in accordance with the ballistic results in terms of spherical bullet penetration into the target. Therefore a phenomenological relationship between strain-rate and compressive strength used for ceramics, which have some points in common with these foams, was adopted. This has been then modified and calibrated on a single impact test speed.

The stress-strain curves obtained by the application of these relationships were then validated on different velocity impact test computations, allowing an high adherence between experimental and numerical simulation results. Additional computations were performed adopting finer mesh combinations of the domain, and showed good consistency of the results.

In conclusion, it is possible to affirm that a simple form model to be employed in numerical simulations, describes accurately high strain rates behaviour of carbon foams under mono-dimensional strain loading conditions.

\section{References}

[1] Gibson, L.J., Ashby, M.F., Cellular solids, structure and Properties, Second Edition, Cambridge (1999).

[2] Oak Ridge National Laboratory (ORNL) Dept. of Energy Website.

[3] M.D. Sarzynski, Carbon Foam Characterization: Sandwich Flexure, Tensile and Shear Response, Master Thesis, (2003).

[4] M. Grujicic, B. Pandurangan, C.L. Zhao, S.B. Biggers, D.R. Morgan, Hypervelocity impact resistance of reinforced carbon-carbon/carbon-foam thermal protection systems, Applied Surface Science, 2005.

[5] J. Lankford, Mechanism Responsible for Strain - Rate - Dependent Compressive Strength in Ceramic Materials, Communications of the American Ceramic Society, (1981).

[6] P. Forquin, A. Arias, R. Zaera, An experimental method of measuring the confined compression strength of high performance concretes to analyse 
their ballistic behaviour. $8^{\text {th }}$ International conference on Mechanical and Physical Behaviour of Materials under Dynamic Loading, J. Cirne, R. Dormeval, et al. J. Phys. IV France 134 (2006) 629-634. DOI: 10.1051/jp4:2006134097.

[7] RILEM TC 148-ssc: Test Methods for compressive softening, 2001.

[8] Ls-Dyna Keyword User's Manual, LSTC, May 2007, Version 971.

[9] Sambamoorthy, Halder, Characterization and Component Level Correlation of Energy Absorbing PU Foams using Ls-Dyna Material Models, Lear Corporation.

[10] Chocron, Walker, Nicholls, Dannemann, Anderson, Analytical model of the confined compression Test Used to Characterize Brittle Materials; Journal of Applied Mechanics, March 2008 vol 75. 